\title{
Metacarpal Bone Digit 4
}

National Cancer Institute

\section{Source}

National Cancer Institute. Metacarpal Bone Digit 4. NCI Thesaurus. Code C52793.

The fourth of five miniature long bones located in the palm of the hand, which articulates proximally with the capitate, hamate, and third and fifth metacarpals at the carpometacarpal joint and distally with the ring finger phalanx at the metacarpophalangeal joint. 